\title{
Mesons in a soft-wall AdS-Schwarzschild approach at low temperature
}

\author{
Thomas Gutsche, ${ }^{1}$ Valery E. Lyubovitskij, ${ }^{1,2,3,4}$ Ivan Schmidt, ${ }^{2}$ and Andrey Yu. Trifonov ${ }^{4}$ \\ ${ }^{1}$ Institut für Theoretische Physik, Universität Tübingen, Kepler Center for Astro and Particle Physics, \\ Auf der Morgenstelle 14, D-72076 Tübingen, Germany \\ ${ }^{2}$ Departamento de Física y Centro Científico Tecnológico de Valparaíso-CCTVal, \\ Universidad Técnica Federico Santa María, Casilla 110-V, Valparaíso, Chile \\ ${ }^{3}$ Department of Physics, Tomsk State University, 634050 Tomsk, Russia \\ ${ }^{4}$ Laboratory of Particle Physics, Tomsk Polytechnic University, 634050 Tomsk, Russia
}

(Received 5 February 2019; published 28 March 2019)

\begin{abstract}
We derive a holographic soft-wall approach in five dimensional AdS-Schwarzschild space for the description of mesons at finite temperature. In this first application we consider the small temperature limit and derive analytical expression for the mass spectrum of mesons with adjustable quantum numbers $n$ (radial number), $L$ (angular orbital momentum) and $J$ (total angular momentum). We explicitly separate the contribution at zero temperature and the leading order temperature correction. The temperature corrections arise from the temperature dependence of the dilaton parameter (which is the parameter of spontaneous breaking of chiral symmetry related to the pseudoscalar meson decay constant) and the warping of the AdS metric due to temperature. We extend our results to any hadron with integer spin (tetraquarks, dibaryons, etc.). We present numerical analysis for the temperature dependence of meson masses and form factors.
\end{abstract}

DOI: 10.1103/PhysRevD.99.054030

\section{INTRODUCTION}

The study of hadron properties at finite temperature is a promising task, since it allows for a deeper understanding of the evolution of the early Universe, the formation of hadronic matter and its phase transitions. Hadrons at finite temperature have been considered in the past in holographic QCD (see, e.g., Refs. [1-13]). In particular, In Ref. [1] it was shown that the deconfinement in the anti-de Sitter approaches of quantum chromodynamics (AdS/QCD) occurs via a first-order Hawking-Page phase transition between low temperature thermal AdS and high temperature black hole. The model-dependent predictions for the deconfinement temperature in the hard (HW) and soft-wall (SW) AdS/QCD have been obtained: $T_{c}^{\mathrm{HW}}=$ $122 \mathrm{MeV}$ and $T_{c}^{\mathrm{SW}}=191 \mathrm{MeV}$, where the $\mathrm{SW}$ prediction was close to the lattice QCD prediction $T_{c}^{\text {Lattice }}=192 \pm$ $7 \pm 4 \mathrm{MeV}$ [14]. A better prediction of the SW AdS/QCD for the deconfinement temperature was related to a more realistic description of meson spectrum in this approach. In Ref. [2] the gravity dual of charmonium in the strongly coupled QCD plasma was constructed on a basis of the

Published by the American Physical Society under the terms of the Creative Commons Attribution 4.0 International license. Further distribution of this work must maintain attribution to the author(s) and the published article's title, journal citation, and DOI. Funded by SCOAP.
SW AdS/QCD model and performing the matching of the ultraviolet behavior of the charm current correlator to the result of QCD. Detailed analysis of the holographic potential forming the charmonium and its spectral function has been presented. In Ref. [3] the free energy of the heavy quark-antiquark pair in strongly interaction matter has been investigated using a holographic approach formulated with the use of the AdS/Reissner-Nordström black-hole metric at finite temperature $T$ and chemical potential $\mu$. The obtained deconfinement line in the $\mu-T$ plane was similar to ones obtained in lattice QCD and other QCD related approaches. In Ref. [4] a SW AdS/QCD approach with the AdS-Schwarzschild geometry has been applied for study the dependence of the chiral condensate on the temperature and quark density. The main finding was that for $\mu$ below a critical value the chiral quark condensate is decreasing with increasing the temperature. In particular, the quark condensate at $\mu=0$ vanishes at $T_{c} \simeq 210 \mathrm{MeV}$. Increasing of $\mu$ leads to decreasing of $T_{c}$. In Ref. [5] spectral functions of the scalar glueball and light vector mesons have been studied in a hot dense medium in a SW AdS/QCD approach based on AdS/Reissner-Nordström metric. It was observed that scalar glueball and vector mesons became unstable at increased values of temperature and chemical potential. Similar analysis of the vector mesons in the SW AdS/QCD approach based using the AdS-Schwarzschild geometry has been performed in Ref. [6]. In Refs. [7] detailed study of thermal properties of charmonium and bottomonium 
vector mesons has been carried out in holographic AdS/QCD The dissociation of heavy vector quarkonia states was studied in the context of the configurational entropy (CE) setup. It was found that $\mathrm{CE}$ has a specific behavior on temperature for charmonium and bottomonium states. In particular, for the charmonium the CE curve increases monotonically and the probability of dissociation of these states in the medium increases with the temperature, while for the bottomonium the picture is different. In case of the bottomonium, the $\mathrm{CE}$ has global minimum at temperatures $T \sim 1.3 T_{c}$ and then for higher temperatures it increases monotonically. In Ref. [8] in-medium properties of mesons have been studied at finite temperature and baryon chemical potential within SW AdS/QCD model with modified dilaton field and holographic potential (by adding the quartic scalar term) in order to obtain the correct form of chiral symmetry breaking and correct spectrum. In particular, it was used a dilaton field parametrization, which is negative quadratic in the ultraviolet limit, while becoming positive quadratic in the infrared region. In Ref. [9] different types of the SW AdS/QCD potentials have been analyzed in order to obtain a melting temperature for different bound states-scalar mesons, glueballs, hybrids, and tetraquarks. One of the main findings was an observation that the melting temperature increases for hadrons containing heavy quarks. In Ref. [10] it was proposed an idea of a thermal dilaton-dilaton field depending on temperature. Two thermal forms of the dilatons have been studied which make it possible to obtain melting temperatures for mesons close to $180 \mathrm{MeV}$. Detailed analysis of the deconfinement temperature for glueballs, scalar and vector mesons in different versions of the HW and SW AdS/QCD approaches has been performed in Ref. [11]. In Ref. [12] the SW AdS/QCD model has been applied for the description of the high- $T_{c}$ superconductivity. In Ref. [13] chiral phase transition has been studied in a SW AdS/QCD model using AdS-Schwarzschild metric and incorporating $S U_{L}\left(N_{f}\right) \times S U_{R}\left(N_{f}\right)$ symmetry. In particular, a detailed analysis of the chiral condensate on temperature has been carried out.

The main motivation for our study is to propose the modification of the soft-wall model at finite temperature in order bring it in consistency with QCD. In particular, we argue that in order to reproduce a temperature behavior of quark condensate one should include temperature dependence of the dilaton field, which is the parameter of spontaneous breaking of chiral symmetry related to the pseudoscalar meson decay constant and the warping of the AdS metric due to temperature. In particular, we propose that the dilaton field has the specific $T$-dependence, which is dictated by the temperature behavior of the chiral quark condensate in QCD $[15,16]$ derived using chiral perturbation theory (ChPT) [17]. In this way we postulate the temperature dependence of the dilaton field using its relation to the chiral quark condensate at zero temperature.
We note that a thermal behavior of the dilaton has been proposed before in Ref. [10], but now in our paper we do it in a way consistent with QCD. It makes such study important to improve understanding hadron properties at finite temperatures.

In the present paper we are interested in the specific low temperature limit and the derivation of analytical formulas for the mass spectrum of mesons and their form factors. In particular, we consider two possible sources of temperature dependence: (1) the warping of the AdS metric due to temperature, (2) the temperature dependence of the dilatonbackground field, which produces confinement and is responsible for the breaking of conformal invariance and the spontaneous breaking of chiral symmetry in holographic QCD.

We consider the propagation of a meson field $M_{J}(x, z)$ with total angular momentum $J$ in five dimensional AdSSchwarzschild space at finite temperature. The AdSSchwarzschild metric is specified by

$$
d s^{2}=e^{2 A(z)}\left[f_{T}(z) d t^{2}-(d \vec{x})^{2}-\frac{d z^{2}}{f_{T}(z)}\right]
$$

where $x=(t, \vec{x})$ is the set of Minkowski coordinates, $z$ is the holographic coordinate, $R$ is the AdS radius and $A(z)=\log (R / z)$. Here $f_{T}(z)=1-z^{4} / z_{H}^{4}$ where $z_{H}$ is the position of the event horizon, which is related to the blackhole Hawking temperature $T=1 /\left(\pi z_{H}\right)$. The latter also represents (holography correspondence) the temperature of the boundary field theory. The holographic coordinate changes from 0 to $z_{H}$. The AdS-Schwarzschild metric breaks conformal invariance because of the temperature dependence. At zero temperature the conformal invariance can be broken by introducing a wall in the $z$-direction. In the following we consider one of the versions of the soft-wall AdS/QCD model [18,19]. It was developed in Refs. [20-23] for the study of mesons, baryons and exotic states with adjustable quantum numbers of total angular spin $J$, angular orbital momentum $L$ and radial quantum number $n$. Following Ref. [18] we introduce the exponential prefactor $\exp [-\varphi(z)]$ in the effective action, containing the background (dilaton) field $\varphi(z)=\kappa^{2} z^{2}$, where $\kappa$ is a scale parameter of the order of a few hundred $\mathrm{MeV}$. This dilaton field breaks conformal invariance, produces confinement and is responsible for the spontaneous breaking of chiral symmetry in holographic QCD. In addition to dilaton we introduce in the action the thermal prefactor

$$
e^{-\lambda_{T}(z)}, \quad \lambda_{T}(z)=\alpha \frac{z^{2}}{z_{H}^{2}}+\gamma \frac{z^{4}}{z_{H}^{4}}+\xi \frac{\kappa^{2} z^{6}}{z_{H}^{4}}
$$

where dimensionless parameters $\alpha, \gamma$, and $\xi$ parametrize the $z^{2}, z^{4}$, and $z^{6}$ thermal corrections. Later we will show that the parameter $\gamma$ is fixed to guarantee the gauge invariance and massless ground-state pseudoscalar mesons $(\pi, K, \eta)$ in 
chiral limit, while the parameter $\xi$ is fixed to drop the radial dependence of six power in the holographic potential in our approach. Also we will demonstrate that the parameter $\alpha$ encodes the contribution of gravity to the restoration of chiral symmetry at a critical temperature $T_{c}$. From our analysis of this phenomena we will find (as will be seen below) that the $\alpha$ parameter increases the $T_{c}$ and its value should be relatively small. A limitation to small $\alpha$ is also consistent with small temperature limit. In the limit $z \ll z_{H}$ the thermal factors $f_{T}(z)$ and $\lambda_{T}(z)$ reduce to 1 , and the AdS-Schwarzschild geometry reduces to the pure AdS case:

$$
d s^{2}=e^{2 A(z)}\left[d t^{2}-(d \vec{x})^{2}-d z^{2}\right] .
$$

Therefore, there are two sources for the breaking of conformal invariance in the soft-wall AdS/QCD model: the dilaton field $\varphi(z)$ and the metric (warping factor $f_{T}(z)$ and prefactor $\left.\exp \left[-\lambda_{T}(z)\right]\right)$.

Recently, in Ref. [10] it was proposed to include an additional temperature dependence of the soft-wall AdS/QCD action via an explicit $T$-dependence of the dilaton field: $\varphi(z) \rightarrow \varphi(z, T)=\kappa^{2}(T) z^{2}$. In particular, two forms for the thermal dilaton were proposed for $\varphi(z, T)$ behavior [10]:

$$
\varphi_{1}(z, T)=\kappa^{2}(1+\alpha T) z^{2}
$$

and

$$
\varphi_{2}(z, T)=\kappa_{1}^{2}(1+\alpha T) z^{2} \tanh \left(\kappa_{2}^{2}(1+\alpha T) z^{2}\right) .
$$

Here we also propose a specific $T$-dependence of the dilaton scaling parameter $\kappa$. We base this choice on the idea that $\kappa^{2}$ as a parameter of spontaneous breaking of chiral symmetry is related in the soft-wall AdS/QCD approach to the quark condensate. At zero temperature one has the definition

$$
\Sigma=\langle 0|\bar{q} q| 0\rangle=-N_{f} B F^{2},
$$

where $N_{f}$ is the number of quark flavors, $B$ is the quark condensate parameter, and $F$ is the pseudoscalar meson decay constant in the chiral limit at zero temperature (e.g., $F \simeq 87 \mathrm{MeV}$ for $N_{f}=2$ [15]). In particular, in the soft-wall AdS/QCD model the dilaton parameter $\kappa$ and the decay constant $F$ are related in the chiral limit at zero temperature as $[19,20]$ :

$$
F=\kappa \frac{\sqrt{3}}{8} .
$$

Substituting Eq. (7) into Eq. (6) we get a relation between $\Sigma$ and $\kappa^{2}$ :

$$
\Sigma=-\frac{3 N_{f} B}{64} \kappa^{2}
$$

We suppose identical temperature dependence of the $\kappa^{2}(T)$ and $\Sigma(T)=\langle 0|\bar{q} q| 0\rangle_{T}$ postulating the relation

$$
\kappa^{2}(T)=\kappa^{2} \frac{\Sigma(T)}{\Sigma} .
$$

Taking into account Eqs. (6) and (7), and the relation

$$
\Sigma(T)=-N_{f} B(T) F^{2}(T)
$$

we can relate $\kappa^{2}(T)$ with $T$-dependent quark condensate parameter $B(T)$ and pseudoscalar coupling constant in the chiral limit $F(T)$ as

$$
\kappa^{2}(T)=\kappa^{2} \frac{B(T)}{B} \frac{F^{2}(T)}{F^{2}}=\frac{64}{3} \frac{B(T)}{B} F^{2}(T) .
$$

In Ref. [15], using two-loop chiral perturbation theory $(\mathrm{ChPT})$, the low-temperature dependence of the quark condensate $\Sigma(T)$ was established:

$$
\begin{aligned}
\Sigma(T) & =\Sigma\left[1-\frac{N_{f}^{2}-1}{N_{f}} \frac{T^{2}}{12 F^{2}}-\frac{N_{f}^{2}-1}{2 N_{f}^{2}}\left(\frac{T^{2}}{12 F^{2}}\right)^{2}+\mathcal{O}\left(T^{6}\right)\right] \\
& =\Sigma\left[1+\Delta_{T}+\mathcal{O}\left(T^{6}\right)\right] .
\end{aligned}
$$

This result is valid for an adjustable number of quark flavors with $N_{f} \geq 2$ and is given as an expansion in $T^{2}$.

The temperature correction to the condensate, up to order $T^{4}$, is encoded in the quantity $\Delta_{T}$ with

$$
\begin{aligned}
\Delta_{T} & =\delta_{T_{1}} \frac{T^{2}}{12 F^{2}}+\delta_{T_{2}}\left(\frac{T^{2}}{12 F^{2}}\right)^{2}, \\
\delta_{T_{1}} & =-\frac{N_{f}^{2}-1}{N_{f}}, \quad \delta_{T_{2}}=-\frac{N_{f}^{2}-1}{2 N_{f}^{2}} .
\end{aligned}
$$

In Ref. [16] the two-loop ChPT result of Ref. [15] has been extended to the three-loop case by the inclusion of the higher-order $\mathcal{O}\left(T^{6}\right)$ term:

$$
\begin{aligned}
\Sigma(T) & =\Sigma\left[1+\tilde{\Delta}_{T}+\mathcal{O}\left(T^{8}\right)\right], \\
\tilde{\Delta}_{T} & =\Delta_{T}+N_{f}\left(N_{f}^{2}-1\right)\left(\frac{T^{2}}{12 F^{2}}\right)^{3} \log \frac{T}{\Lambda_{\Sigma}} .
\end{aligned}
$$

The scale $\Lambda_{\Sigma}$ absorbs the ultraviolet divergencies in the three-loop graphs, generated by the leading term $L_{\mathrm{ChPT}}^{(2)}$ in the ChPT Lagrangian. The value of $\Lambda_{\Sigma}$ can been fixed by the low-energy constants in the next-to-leading ChPT Lagrangian $\mathcal{L}_{\text {ChPT }}^{(4)}$ using data. E.g., in the two-flavor case $\Lambda_{\Sigma}$ has been related to the $D$-wave isospin zero $\pi \pi$ scattering length $a_{2}^{0}$, leading to $\Lambda_{\Sigma}=470 \pm 110 \mathrm{MeV}$. 
Here we restrict to an accuracy of $\mathcal{O}\left(T^{4}\right)$ in the temperature expansion of the quark condensate. Therefore, using the relations (9) and (12) we obtain the $T$-dependence of the dilaton field with

$$
\varphi(z, T)=\kappa^{2}(T) z^{2}, \quad \kappa^{2}(T)=\kappa^{2}\left[1+\Delta_{T}+\mathcal{O}\left(T^{6}\right)\right] .
$$

In the following it is useful to combine the two terms, the $T$-dependent dilaton field and the $z^{2}$ term in the thermal prefactor $e^{-\lambda_{T}(z)}(2)$ as

$$
\varphi(z, T)+\frac{\alpha z^{2}}{z_{H}^{2}}=K_{T}^{2} z^{2},
$$

where

$$
\begin{aligned}
K_{T}^{2} & =\kappa^{2}(T)+\frac{\alpha}{z_{H}^{2}}=\left(1+\rho_{T}\right) \kappa^{2}, \\
\rho_{T} & =\left(\frac{9 \alpha \pi^{2}}{16}+\delta_{T_{1}}\right) \frac{T^{2}}{12 F^{2}}+\delta_{T_{2}}\left(\frac{T^{2}}{12 F^{2}}\right)^{2}+\mathcal{O}\left(T^{6}\right) .
\end{aligned}
$$

Note the $T$-dependence of $B(T)$ and $F(T)$ has been studied in Refs. [15] and [24]. In particular, $F(T)$ was calculated at one-loop in Ref. [15]

$$
F(T)=F\left[1-\frac{N_{f}}{2} \frac{T^{2}}{12 F^{2}}+\mathcal{O}\left(T^{4}\right)\right]
$$

and at the level of two loops for $N_{f}=2$ in Ref. [24]

$$
F^{2}(T)=F^{2}\left[1-\frac{T^{2}}{6 F^{2}}+\frac{T^{4}}{36 F^{4}} \log \frac{\Lambda_{F}}{T}+\mathcal{O}\left(T^{6}\right)\right],
$$

where $\Lambda_{F}=2.3 \mathrm{GeV}$ is the scale absorbing the ultraviolet divergencies.

In the finite temperature case it is useful to introduce the Regge-Wheeler (RW) tortoise coordinate $r^{*}$ instead of the holographic variable $z$ via the substitution $[25,26]$ :

$$
r^{*}=-\int \frac{d z}{f_{T}(z)}=\frac{z_{H}}{2}\left[-\arctan \frac{z}{z_{H}}+\frac{1}{2} \log \frac{1-z / z_{H}}{1+z / z_{H}}\right] .
$$

For convenience we will use the variable $r=-r^{*}$. Note that in the low temperature limit the $r$ coordinate can be expanded as

$$
r=z\left[1+\frac{t^{4}}{5}+\frac{t^{8}}{9}+\mathcal{O}\left(t^{12}\right)\right], \quad t=z / z_{H} .
$$

The holographic coordinate, expanded in powers of $r$, is

$$
z=r\left[1-\frac{t_{r}^{4}}{5}+\frac{4}{45} t_{r}^{8}+\mathcal{O}\left(t_{r}^{12}\right)\right], \quad t_{r}=r / z_{H}
$$

In order of accuracy, $\mathcal{O}\left(T^{4}\right)$, we are working we restrict to the leading-order (LO) and next-to-leading-order (NLO) term in the expansion of $z$ in Eq. (22). In this case the metric is

$$
\begin{gathered}
d s^{2}=e^{2 A(r)} f_{T}^{3 / 5}(r)\left[d t^{2}-\frac{d \vec{x}^{2}}{f_{T}(r)}-d r^{2}\right], \\
A(r)=\log (R / r), \quad f_{T}(r)=1-r^{4} / z_{H}^{4} .
\end{gathered}
$$

The product of the prefactors containing dilaton field and thermal factor $\lambda_{T}$ in terms of the $r$ variable is

$$
\mathcal{P}=\exp \left[-\varphi_{T}(r)-\gamma \frac{r^{4}}{z_{H}^{4}}-\frac{\kappa^{2} r^{6}}{z_{H}^{4}}\left(\xi-\frac{2}{5}\right)\right],
$$

where $\varphi_{T}(r)$ is the dilaton field

$$
\varphi_{T}(r)=K_{T}^{2} r^{2}=\left(1+\rho_{T}\right) \kappa^{2} r^{2} .
$$

In order to suppress the $r^{6}$ terms, which could later on enter to the holographic potential defining the properties of hadrons, we fix the parameter $\xi$ as $\xi=2 / 5$. The parameter $\gamma$ is fixed to guarantee the gauge invariance and massless ground-state pseudoscalar mesons $(\pi, K, \eta)$ in chiral limit, i.e., it should provide that $r^{4}$ terms are vanish for $J=0$ and $J=1$ and contribute only for higher spins states $J \geq 2$. It will be fixed fixed later (in next section) in the consideration of the equation of motion for the AdS bulk profile. After fixing the $\xi$ parameter, the prefactor $\mathcal{P}$ reads

$$
\mathcal{P}=\exp \left[-\varphi_{T}(r)-\gamma \frac{r^{4}}{z_{H}^{4}}\right] .
$$

In Eqs. (24) and (26) we drop higher-order temperature dependent terms $\mathcal{O}\left(T^{6}\right)$.

From Eq. (17) we can fix the critical temperature $T_{c}$ in the soft-wall AdS/QCD model which corresponds to a vanishing dilaton parameter $K_{T_{c}}^{2}=0$. Note that in comparison with ChPT we have an additional piece contributing to chiral restoration, which comes from the warping thermal factor induced by gravity. We expect that this additional gravitational effect must be perturbative and does not significantly change the QCD prediction. In particular, QCD based on the two-loop ChPT calculation (12) predicts the following result for the critical temperature at order $T^{4}$

$$
\frac{\left(T_{c}^{\mathrm{QCD}}\right)^{2}}{12 F^{2}}=N_{f}\left[\sqrt{\frac{N_{f}^{2}+1}{N_{f}^{2}-1}}-1\right] .
$$


$T_{c}^{\mathrm{QCD}}$ has the power scaling behavior $T_{c}^{\mathrm{QCD}} \sim F / \sqrt{N_{f}}$ at large $N_{f}$. The thermal warping factor changes the QCD prediction for $T_{c}$ to

$$
\frac{T_{c}^{2}}{12 F^{2}}=N_{f}\left[\sqrt{\frac{N_{f}^{2}+1}{N_{f}^{2}-1}-2 \beta+\beta^{2}}-1+\beta\right],
$$

where

$$
\beta=\frac{9 \alpha \pi^{2}}{16} \frac{N_{f}}{N_{f}^{2}-1}
$$

At large $N_{f}$ and small $\alpha, T_{c}$ can be expanded as

$$
T_{c}=2 F \sqrt{\frac{3}{N_{f}}}\left[1+\frac{9 \pi^{2}}{32} \frac{\alpha}{N_{f}}+\mathcal{O}\left(1 / N_{f}^{2}\right)\right] .
$$

In Fig. 1 we plot the dependence of the quantity $R_{c}=$ $T_{c}^{2} /\left(12 F^{2}\right)$ on the number of quark flavors $N_{f}$ and the coupling $\alpha$. One can see that $R_{c}$ decreases when $N_{f}$ increases, while it increases with a growth of the parameter $\alpha . R_{c}$ rapidly grows with increasing $\alpha$ for $N_{f}=2$. In Figs. 2-5 we present the 3 dimensional plots for the $T$-dependence of the dilaton parameter $K_{T}^{2}$ for four cases: (1) $N_{f}=2, F=87 \mathrm{MeV}$, (2) $N_{f}=3, F=100 \mathrm{MeV}$, (3) $N_{f}=4, F=130 \mathrm{MeV}$, and (4) $N_{f}=5, F=140 \mathrm{MeV}$. In each case the $T_{c}$ regime corresponds to the intersection of the 3 dimensional plot with the $(T, \alpha)$ plane. The critical temperature for all number of flavors is enhanced when increasing the parameter $\alpha$. In Table I we present numerical results for the critical temperature for different number of flavors and for three specific values of $\alpha=0,0.1,0.2$. One can see that the $\alpha$ parameter increases the $T_{c}$ and its value should be relatively small in order exclude its big contribution to the shift of the value of the $T_{c}$. Note, due to expansion of the chiral condensate in

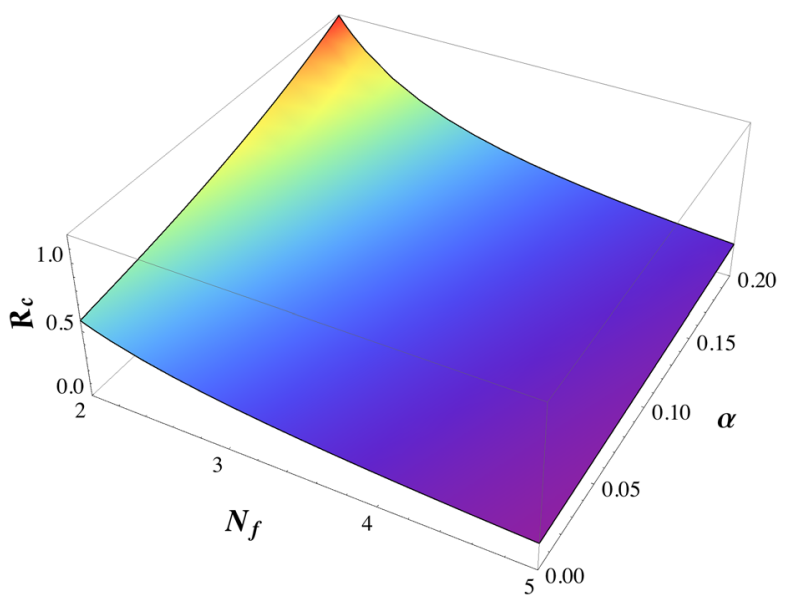

FIG. 1. Dependence of the critical temperature (contained in $R_{c}=T_{c}^{2} / 12 F^{2}$ ) on the number of flavors $N_{f}$ and the parameter $\alpha$.

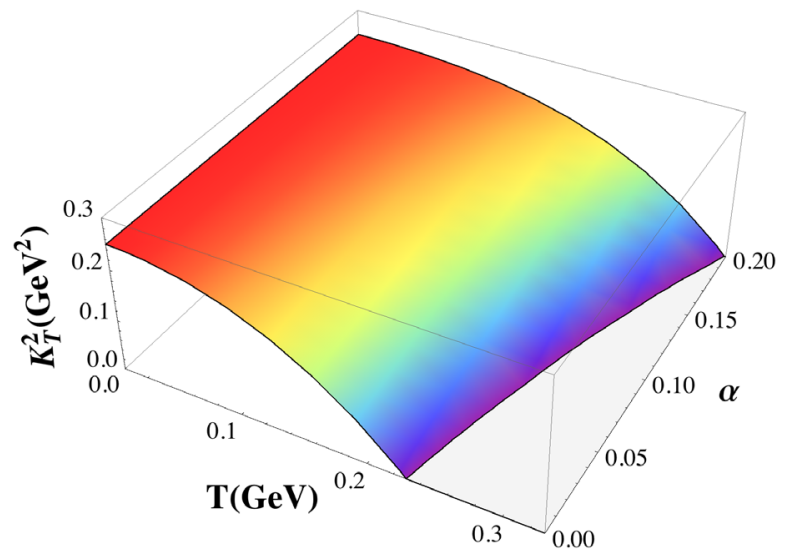

FIG. 2. $\quad K_{T}^{2}$ for $N_{f}=2$ and $F=87 \mathrm{MeV}$.

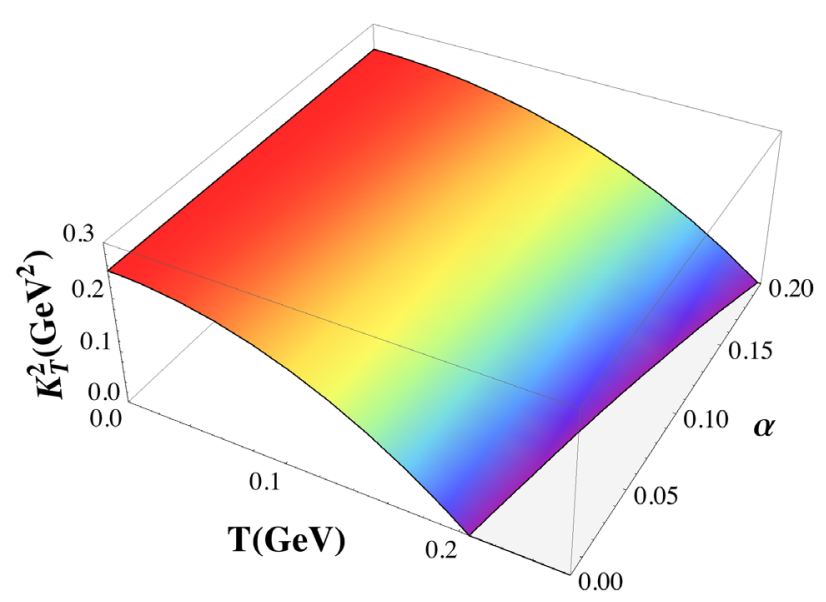

FIG. 3. $K_{T}^{2}$ for $N_{f}=3$ and $F=100 \mathrm{MeV}$.

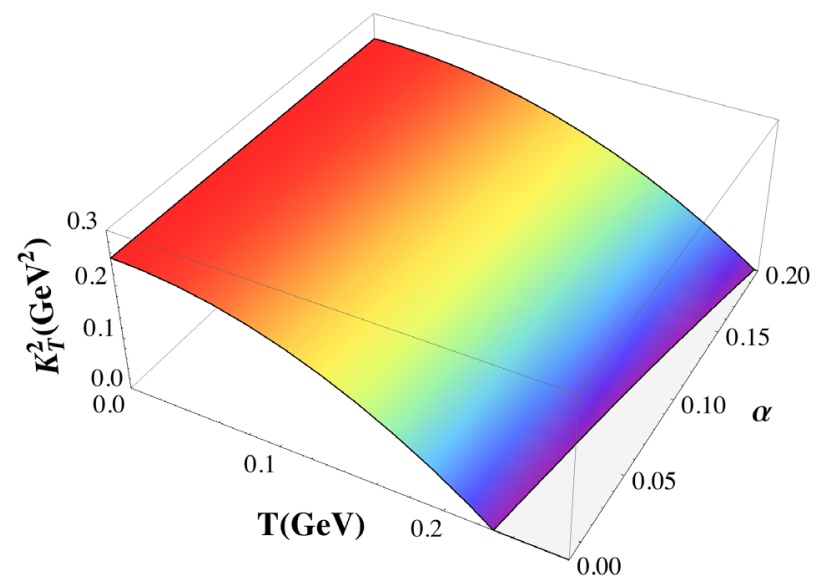

FIG. 4. $K_{T}^{2}$ for $N_{f}=4$ and $F=130 \mathrm{MeV}$.

powers of $T^{2} /\left(12 F^{2}\right)$ the analysis at small temperature can be valid at $T<2 F \sqrt{3} \simeq 300-500 \mathrm{MeV}$ in case of number of flavors varied from 2 to 5 .

After these preliminaries we can formulate our approach, starting from an effective action at finite temperature and then considering the applications to the mass spectrum and 


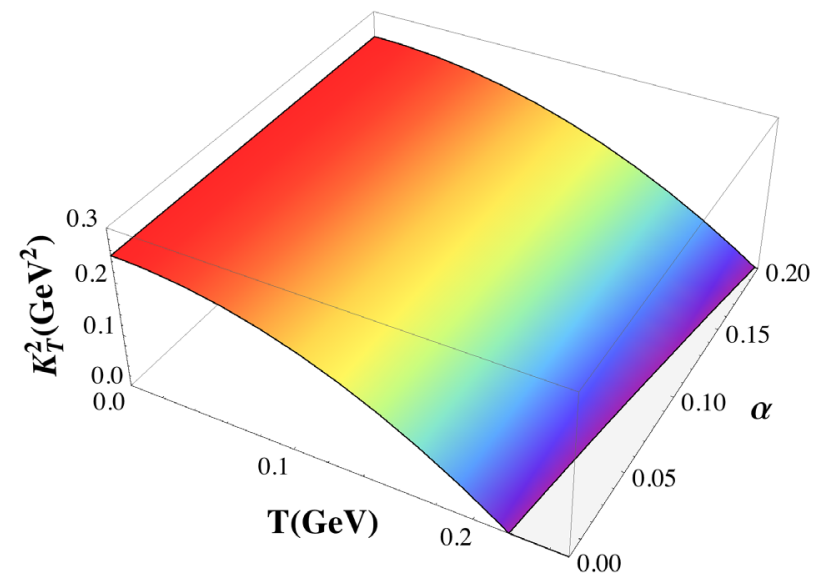

FIG. 5. $K_{T}^{2}$ for $N_{f}=5$ and $F=140 \mathrm{MeV}$.

the form factors of mesons. The paper is structured as follows. In Sec. II we present the details for the construction of an effective action at small temperatures and apply it to the calculation of the mass spectrum and form factors of hadrons with integer spin $J$ (mesons, tetraquarks, dibaryons, etc.). In Sec. III we present numerical results for the
TABLE I. Dependence of $T_{c}$ on $N_{f}$ and $\alpha$.

\begin{tabular}{llll}
\hline \hline & \multicolumn{3}{c}{$T_{c}(\mathrm{MeV})$} \\
\cline { 2 - 4 }$N_{f}$ & $\alpha=0$ & $\alpha=0.1$ & $\alpha=0.2$ \\
\hline 2 & 230 & 270 & 329.3 \\
3 & 206.1 & 228.2 & 258 \\
4 & 228.9 & 246.6 & 268.8 \\
5 & 219.1 & 232.4 & 248.3 \\
\hline \hline
\end{tabular}

mass spectrum and the form factors of mesons. Finally, in Sec. IV, we summarize the results of the paper.

\section{FRAMEWORK}

\section{A. Effective action and hadron masses at low temperatures}

In this section we start with the derivation of a five dimensional action for the boson bulk field $M_{N_{1} \ldots N_{J}}(x, r, T)$, with arbitrary total integer spin $J$ at small temperature $T$. Our formalism is based on the analogous action at zero temperature [20] and includes the issues discussed in the previous section. The action reads:

$$
\begin{aligned}
S_{M}= & \frac{(-)^{J}}{2} \int d^{4} x d r \sqrt{g} e^{-\varphi_{T}(r)-\gamma r^{4} / z_{H}^{4}}\left[\partial_{N} M_{N_{1} \ldots N_{J}}(x, r, T) \partial^{N} M^{N_{1} \ldots N_{J}}(x, r, T)\right. \\
& \left.-\left(\mu_{J}^{2}(r, T)+V_{J}(r, T)\right) M_{N_{1} \ldots N_{J}}(x, r, T) M^{N_{1} \ldots N_{J}}(x, r, T)\right]
\end{aligned}
$$

where $(x, r)$ is the set of four Minkowski and holographic coordinates. The dilaton $\varphi_{T}(r)$ and metric $d s^{2}$ are specified in Eqs. (25) and (23), $\sqrt{g}=(R / r)^{5}$, and

$V_{J}(r, T)=\frac{e^{-2 A(r)}}{f_{T}^{3 / 5}(r)}\left[\varphi_{T}^{\prime \prime}(r)+(3-2 J) \varphi_{T}^{\prime}(r) A^{\prime}(r)\right]$

is the dilaton potential with $F^{\prime}(r)=d F(r) / d r, F^{\prime \prime}(r)=$ $d^{2} F(r) / d r^{2}$ and $F=\varphi, A$.

The quantity $\mu_{J}^{2}(r, T)$ is the bulk boson mass at finite temperature, which is related to the bulk boson mass at zero temperature $\mu_{J}^{2}$ as

$$
\mu_{J}^{2}(r, T)=\frac{\mu_{J}^{2}}{f_{T}^{3 / 5}(r)}
$$

As is known, the $\mu_{J}^{2}$ is expressed in terms of the dimension $(\Delta)$ of the interpolating operator dual to the spin- $J$ bulk boson field as

$$
\mu_{J}^{2} R^{2}=(\Delta-J)(\Delta+J-4) .
$$

We therefore have

$$
\mu_{J}^{2}(r, T) R^{2}=\frac{1}{f_{T}^{3 / 5}(r)}(\Delta-J)(\Delta+J-4) .
$$

For the case of bulk fields dual to the $N$-partonic state we get $\Delta=N+L$, where $L=\max \left|L_{z}\right|$ is the maximal value of the $z$-component of the quark orbital angular momentum in the light-front wave function [19]. For mesons, tetraquarks, and sixquarks/dibaryons we have $N=2,4$, and 6 , respectively.

Using the axial gauge $M_{z}(x, r, T)=0$ we perform a Kaluza-Klein expansion for the four-dimensional transverse components of the AdS fields

$$
M_{\mu_{1} \ldots \mu_{J}}(x, r, T)=\sum_{n} M_{\mu_{1} \ldots \mu_{J}, n}(x) \Phi_{n J}(r, T),
$$

where $n$ is the radial quantum number and $M_{\mu_{1} \ldots \mu_{J}, n}(x)$ is the tower of the Kaluza-Klein (KK) modes dual to mesons with spin $J . \Phi_{n J}(r, T)$ are their extra-dimensional profiles (wave functions) depending on the temperature.

After straightforward calculations [21] one can derive the Schrödinger-type equation of motion for the profile $\phi_{n J}(r, T)=e^{-B_{T}(r) / 2} \Phi_{n J}(r, T)$ with 
$B_{T}(r)=\varphi_{T}(r)+A(r)(2 J-3)+\frac{r^{4}}{5 z_{H}^{4}}(2 J-3+5 \gamma)$.

Here we can fix the parameter $\gamma$. In order to suppress the contribution of the $r^{4}$ term in the holographic potential to the mass spectrum of pseudoscalar ground state mesons $(\pi, K, \eta)$ and to the bulk-to-boundary propagator of the $J=1$ vector fields to guarantee the charge conservation we can fix $5 \gamma=J(J-3)+3$. As result the $B_{T}(r)$ reads

$$
B_{T}(r)=\varphi_{T}(r)+A(r)(2 J-3)+\frac{r^{4}}{5 z_{H}^{4}} J(J-1)
$$

and at $J=0$ and $J=1$ the $r^{4}$ term vanishes. In the rest frame of the AdS field with $\vec{p}=0$ we get

$$
\left[-\frac{d^{2}}{d r^{2}}+U_{J}(r, T)\right] \phi_{n J}(r, T)=M_{n J}^{2}(T) \phi_{n J}(r, T),
$$

where $U_{J}(r, T)$ is the effective potential at finite temperature, which can be decomposed into a zero temperature term $U_{J}(r) \equiv U_{J}(r, 0)$ and a temperature dependent term $\Delta U_{J}(r, T)$

$$
\begin{aligned}
U_{J}(r, T)= & U_{J}(r)+\Delta U_{J}(r, T), \\
U_{J}(r)= & \kappa^{4} r^{2}+2 \kappa^{2}(J-1)+\frac{4 m^{2}-1}{4 r^{2}}, \\
\Delta U_{J}(r, T)= & 2 \rho_{T} \kappa^{2}\left(\kappa^{2} r^{2}+J-1\right) \\
& +\frac{4 r^{2}}{5 z_{H}^{4}} J(J-1)\left(\kappa^{2} r^{2}-J\right),
\end{aligned}
$$

where $m=N+L-2$.

At zero temperature $T=0$ the Schrödinger-type EOM

$$
\left[-\frac{d^{2}}{d r^{2}}+U_{J}(r, 0)\right] \phi_{n J}(r, 0)=M_{n J}^{2}(r, 0) \phi_{n J}(r, 0)
$$

has analytical solutions. The resulting wave function

$$
\phi_{n J}(r, 0)=\sqrt{\frac{2 \Gamma(n+1)}{\Gamma(n+m+1)}} \kappa^{m+1} r^{m+1 / 2} e^{-\kappa^{2} r^{2} / 2} L_{n}^{m}\left(\kappa^{2} r^{2}\right)
$$

corresponds to the mass spectrum

$$
M_{n J}^{2}(0)=4 \kappa^{2}\left(n+\frac{m+J}{2}\right)
$$

of the bosonic hadrons composed of $N$ constituents with spin $J$, angular orbital momentum $L$, and the radial quantum number $n$. Here we use the generalized Laguerre polynomials

$$
L_{n}^{m}(x)=\frac{x^{-m} e^{x}}{n !} \frac{d^{n}}{d x^{n}}\left(e^{-x} x^{m+n}\right) .
$$

Temperature corrections to hadronic mass spectrum are evaluated perturbatively considering hadronic wave functions at $T=0$ as unperturbed solutions:

$$
\begin{aligned}
\Delta M_{n J}^{2}(T) & =\left\langle\phi_{n J}(0)\left|\Delta U_{J}(T)\right| \phi_{n J}(0)\right\rangle \\
& =\int_{0}^{\infty} d r \phi_{n J}^{2}(r, 0) \Delta U_{J}(r, T) .
\end{aligned}
$$

In the low temperature case the hadronic mass spectrum is

$$
\begin{aligned}
M_{n J}^{2}(T)= & M_{n J}^{2}(0)+\Delta M_{n J}^{2}(T), \\
\Delta M_{n J}^{2}(T)= & \rho_{T} M_{n J}^{2}(0)+R_{n J} \frac{\pi^{4} T^{4}}{\kappa^{2}}, \\
R_{n J}= & \frac{4}{5} J(J-1)[(m+1)(m+2) \\
& +(6 n-J)(n+m+1)-n J] .
\end{aligned}
$$

The solution for the bulk profile $\phi_{n J}(r, T)$ reads

$\phi_{n J}(r, T)=\sqrt{\frac{2 \Gamma(n+1)}{\Gamma(n+m+1)}} K_{T}^{m+1} r^{m+1 / 2} e^{-K_{T}^{2} r^{2} / 2} L_{n}^{m}\left(K_{T}^{2} r^{2}\right)$.

Note that the normalizable modes $\Phi_{n J}(r, T)$ and $\phi_{n J}(r, T)$ obey the following normalization conditions:

$$
\begin{aligned}
\int_{0}^{\infty} d r e^{-B_{T}(r)} \Phi_{m J}(r, T) \Phi_{n J}(r, T) & =\int_{0}^{\infty} d r \phi_{m J}(r, T) \phi_{n J}(r, T) \\
& =\delta_{m n}
\end{aligned}
$$

The mode $\Phi_{n}(r, T)$ has the correct behavior in both the ultraviolet (UV) and infrared (IR) limits:

$$
\begin{aligned}
& \Phi_{n J}(r, T) \sim r^{N+L-J} \text { at small } r, \\
& \Phi_{n J}(r, T) \rightarrow 0 \text { at large } r .
\end{aligned}
$$

The above formulas are valid for any set of quantum numbers $(n, L, J)$ and number of constituents $N$.

Finally, in this section we include the effects of finite quark masses for low temperatures. Following the ideas developed in our previous papers $[22,23]$ we can readily do it for light mesons and tetraquarks. In particular, taking into account the $T$-dependence of the light quark condensate parameter $B(T)$, we derive the following LO quark mass corrections:

$$
\delta M_{\pi}^{2}(T)=2 \hat{m} B(T)
$$


for the pion and

$$
\delta M_{K}^{2}(T)=\left(\hat{m}+m_{s}\right) B(T)
$$

for the kaon, where $\hat{m}=\left(m_{u}+m_{d}\right) / 2$ is the average mass of $u$ and $d$ quarks, $m_{s}$ is the strange quark mass. The $T$-dependence of $B(T)$ is defined by Eqs. (10), (18), and (19).

Light nonstrange $T_{4 q}$, single strange $T_{3 q s}$, double strange $T_{2 q 2 s}$, single nonstrange $T_{3 s q}$, and strange tetraquark $T_{4 s}$ masses get corrections, which are simply expressed in terms of the pion and kaon masses corrections as [23]:

$$
\begin{aligned}
\delta M_{T_{4 q}}^{2} & \equiv 2 \delta M_{\pi}^{2}, \quad \delta M_{T_{3 q s}}^{2} \equiv \delta M_{\pi}^{2}+\delta M_{K}^{2} \\
\delta M_{T_{2 q 2 s}}^{2} & \equiv 2 \delta M_{K}^{2}, \quad \delta M_{T_{3 s q}}^{2} \equiv 3 \delta M_{K}^{2}-\delta M_{\pi}^{2} \\
\delta M_{T_{4 s}}^{2} & \equiv 4 \delta M_{K}^{2}-2 \delta M_{\pi}^{2} .
\end{aligned}
$$

\section{B. Hadron form factors at low temperatures}

In this section we derive the results for the form factors of hadrons with integer spin (mesons, tetraquarks, sixquarks/dibaryons, etc.) at low temperature. Following our study in Ref. [21], we calculate hadron form factors at low temperatures, induced by the coupling of AdS fields dual to hadrons with external vector AdS fields dual to the electromagnetic field. First, we calculate the vector bulk-toboundary propagator at low temperatures using the universal action derived in Eq. (31). The corresponding EOM for the Fourier transform of the bulk-to-boundary propagator $V(Q, r, T)$, in Euclidean metric $Q^{2}=-q^{2}$, reads:

$$
\partial_{r}\left(\frac{e^{-\varphi_{T}(r)}}{r} \partial_{r} V(Q, r, T)\right)-Q^{2} \frac{e^{-\varphi_{T}(r)}}{r} V(Q, r, T)=0 .
$$

This EOM is similar to the EOM for the case of zero temperature and the only difference is that the temperature dependence is absorbed in the $T$-dependence of the dilaton parameter. Therefore, the solution for the bulk-to-boundary propagator at small temperature is straightforward [27]:

$$
\begin{aligned}
V(Q, r, T) & =\Gamma\left(1+a_{T}\right) U\left(a_{T}, 0, K_{T}^{2} r^{2}\right) \\
& =K_{T}^{2} r^{2} \int_{0}^{1} \frac{d x}{(1-x)^{2}} x^{a_{T}} e^{-K_{T}^{2} r^{2} \frac{x}{1-x}} \\
a_{T} & =\frac{Q^{2}}{4 K_{T}^{2}}
\end{aligned}
$$

where $\Gamma(n)$ and $U(x, y, z)$ are the gamma and Tricomi function, respectively. Now we can calculate the form factor $F_{n J}\left(Q^{2}, T\right)$ depending on the Euclidean momentum squared $Q^{2}$ for bosonic hadrons with quantum numbers $(n, J, L)$ and number of constituents $N$ at low temperature. The master formula is

$$
F_{n J}\left(Q^{2}, T\right)=\int_{0}^{\infty} d r \phi_{n J}^{2}(r, T) V(Q, r, T)
$$

Note that at finite temperature the form factor $F_{n J}\left(Q^{2}, T\right)$ is properly normalized with $F_{n J}(0, T)=1$ because of $V(0, r, T)=1$ and $\int_{0}^{\infty} d r \phi_{n J}^{2}(r, T)=1$. Also, $F_{n J}\left(Q^{2}, T\right)$ has the correct power scaling at large $Q^{2}$, consistent with quark counting rules and independent of the quantum numbers $n$ and $J$, while depending on the number of constituents $N$ and the orbital momentum $L$ :

$$
F_{n J}\left(Q^{2}\right) \sim \frac{1}{\left(Q^{2}\right)^{m+1}}
$$

Using Eqs. (42) and (54) we get for the ground state $n=0$ meson

$$
F_{0 J}\left(Q^{2}, T\right)=\frac{\Gamma\left(a_{T}+1\right) \Gamma(m+2)}{\Gamma\left(a_{T}+m+2\right)} .
$$

Results for radial excitations with any value for $n$ are readily obtained. For example, for the first two radial excitations $n=1$ and $n=2$ the form factors are

$$
\begin{aligned}
F_{1 J}\left(Q^{2}, T\right)= & \frac{\Gamma\left(a_{T}+1\right) \Gamma(m+4)}{\Gamma\left(a_{T}+m+4\right)} \\
& +a_{T}(m+1) \frac{\Gamma\left(a_{T}+2\right) \Gamma(m+2)}{\Gamma\left(a_{T}+m+4\right)},
\end{aligned}
$$

$$
\begin{aligned}
F_{2 J}\left(Q^{2}, T\right)= & \frac{\Gamma\left(a_{T}+1\right) \Gamma(m+6)}{\Gamma\left(a_{T}+m+6\right)} \\
& +a_{T} \frac{\Gamma\left(a_{T}+2\right) \Gamma(m+3)}{\Gamma\left(a_{T}+m+6\right)} \\
& \times\left[(m+5)(2 m+3)+\frac{1}{2}(m+1) a_{T}\left(a_{T}+5\right)\right] .
\end{aligned}
$$

Next we can perform a small $T$-expansion of the form factors. For the ground state form factor we get

$$
\begin{aligned}
F_{0 J}\left(Q^{2}, T\right)= & F_{0 J}\left(Q^{2}\right)+\Delta F_{0 J}\left(Q^{2}\right), \\
F_{0 J}\left(Q^{2}, 0\right)= & \frac{\Gamma(a+1) \Gamma(m+2)}{\Gamma(a+m+2)}, \\
\Delta F_{0 J}\left(Q^{2}, T\right)= & \rho_{T} a \frac{\Gamma(a+1) \Gamma(m+2)}{\Gamma(a+m+2)} \\
& \times[\psi(a+m+2)-\psi(a+1)],
\end{aligned}
$$

where $a=Q^{2} /\left(4 \kappa^{2}\right)$ and $\psi(n)=\Gamma^{\prime}(n) / \Gamma(n)$ is the polygamma function. 


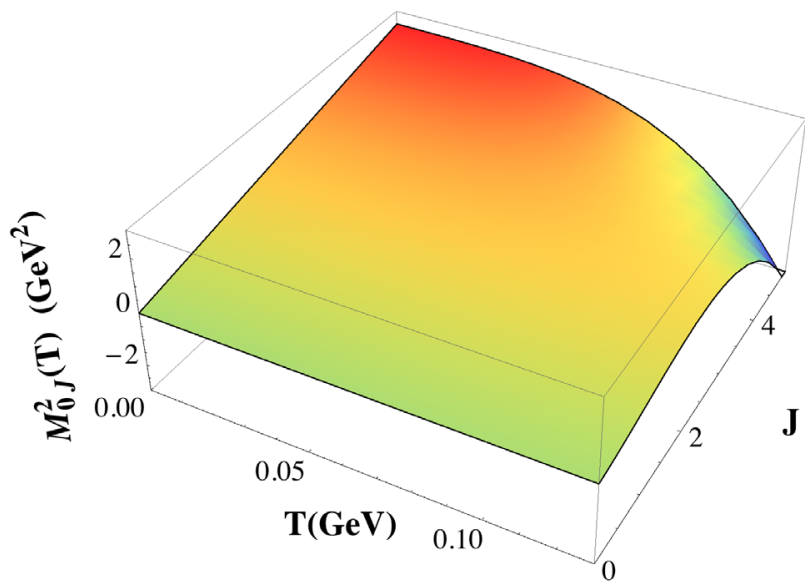

(a)

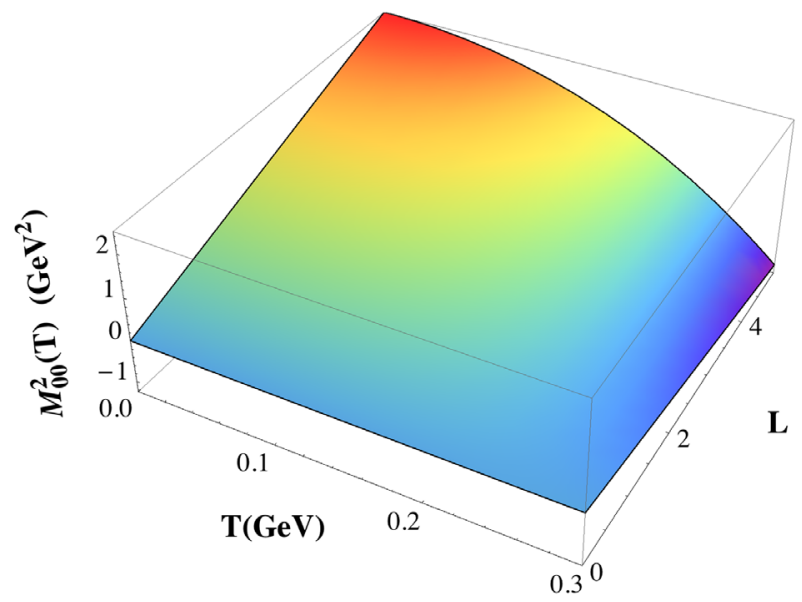

(b)

FIG. 6. $T$-dependence of meson masses: (a) $M_{0 J}^{2}(T)$ for $L=0$ and for $J=0, \ldots, 5$; (b) $M_{00}^{2}(T)$ for $J=0$ and for $L=0, \ldots, 5$.

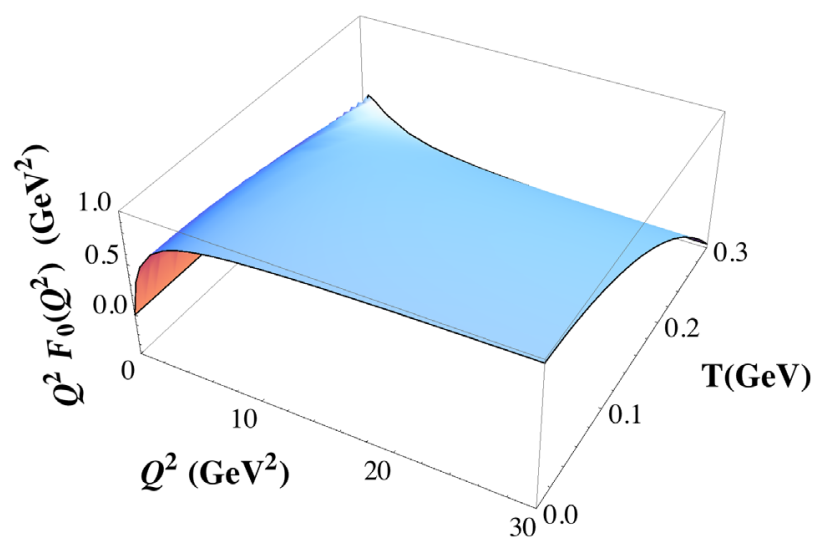

FIG. 7. Temperature dependence of meson form factor multiplied by $Q^{2}$ for $n=0, L=0$, and $J=0$.

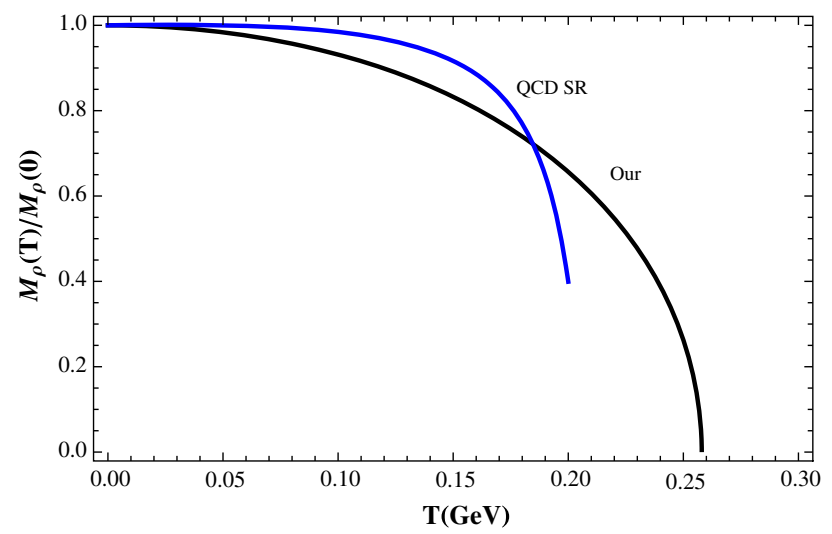

FIG. 8. Comparison of $M_{\rho}(T) / M_{\rho}(0)$ with result of QCD sum rules [28].

\section{NUMERICAL APPLICATIONS}

In this section we present our numerical results for the mass spectrum and form factors of mesons at small temperatures and a particular choice for the parameter $\alpha=0.2$ in the dilaton parameter $K_{T}^{2}$ (17). In Fig. 6 we show our results for the temperature dependence of meson masses: (a) $M_{0 J}^{2}(T)$ for $n=0, L=0$ and for continuous total spin $J$ running from 0 to 5 and (b) $M_{00}^{2}(T)$ for $n=0$, $J=0$, and for continuous orbital angular momentum $L$ running from 0 to 5 . The temperature dependence of meson form factor multiplied by $Q^{2}$ for $n=0, L=0$, and $J=0$ is shown in Fig. 7. A comparison for the $T$ dependence of our results for the $\rho$ meson mass and pion form factor $F_{\pi}\left(Q^{2}\right)$ with results of QCD sum rules calculations [28,29] is given in Figs. 8 and 9. We use normalizations $M_{\rho}(T) / M_{\rho}(0)$ and $F_{\pi}\left(Q^{2}, T\right) / F_{\pi}\left(Q^{2}, 0\right)$ for $Q^{2}=3 \mathrm{GeV}^{2}$.

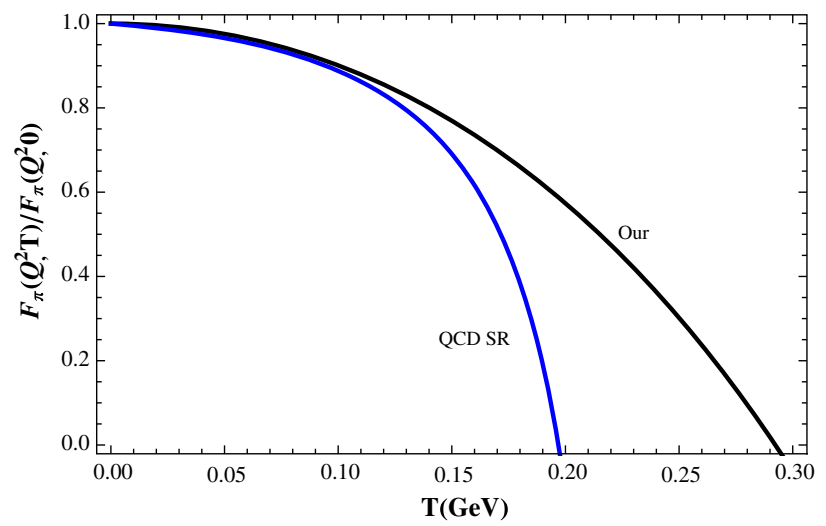

FIG. 9. Comparison of $F_{\pi}\left(Q^{2}, T\right) / F_{\pi}(0, T)$ with result of QCD sum rules [29] for $Q^{2}=3 \mathrm{GeV}^{2}$. 


\section{SUMMARY}

We have proposed a soft-wall AdS/QCD model which implements important features of QCD at zero and low temperatures. In particular, we showed that the dilaton field, being responsible for spontaneous breaking of chiral and conformal symmetry, plays an important role in the temperature dependence of hadronic properties. The $T$-dependence coincides with the one of the quark condensate in QCD. In addition to the dilaton we introduce in the action the thermal prefactor $e^{-\lambda_{T}(z)}$, where the thermal function $\lambda_{T}(z)$ contains $z^{2}, z^{4}$, and $z^{6}$ terms. The $z^{4}$ term guarantees the gauge invariance and massless ground-state pseudoscalar mesons in the chiral limit. The $z^{6}$ term guarantees the absence of power-six terms in the holographic potential. The quadratic $z^{2}$ term gives perturbative contribution to the dilaton field and to the restoration of chiral symmetry at a critical temperature $T_{c}$. Combining the dilaton field with the $z^{2}$ term in the $\lambda_{T}(z)$ thermal function, we introduced the generalized temperature dependent dilaton field. As a consequence the thermal behavior of the generalized dilaton is dominated by a QCD piece, plus a perturbative term due to the $z^{2}$ thermal prefactor. Using the QCD prediction for the small $T$-dependence of the QCD condensate, we predict the thermal behavior of masses and form factors of hadrons with integer total angular momentum $J$. We present numerical results for the critical temperature (when the dilaton field is vanishing), and for the $T$-dependence of masses and form factors of mesons.

\section{ACKNOWLEDGMENTS}

This work was funded by the Carl Zeiss Foundation under Project "Kepler Center für Astro- und Teilchenphysik: Hochsensitive Nachweistechnik zur Erforschung des unsichtbaren Universums (Gz: 0653-2.8/581/2)", by CONICYT (Chile) under Grants No. 7912010025, No. 1180232 and PIA/Basal FB0821, by the Russian Federation program "Nauka" (Contract No. 3.6832.2017/ 8.9), by Tomsk State University competitiveness improvement program under Grant No. 8.1.07.2018, and by Tomsk Polytechnic University Competitiveness Enhancement Program (Grant No. VIU-FTI-72/2017).
[1] C. P. Herzog, Phys. Rev. Lett. 98, 091601 (2007).

[2] H. R. Grigoryan, P. M. Hohler, and M. A. Stephanov, Phys. Rev. D 82, 026005 (2010).

[3] P. Colangelo, F. Giannuzzi, and S. Nicotri, Phys. Rev. D 83, 035015 (2011).

[4] P. Colangelo, F. Giannuzzi, S. Nicotri, and V. Tangorra, Eur. Phys. J. C 72, 2096 (2012).

[5] P. Colangelo, F. Giannuzzi, and S. Nicotri, J. High Energy Phys. 05 (2012) 076.

[6] L. A. H. Mamani, A. S. Miranda, H. Boschi-Filho, and N. R. F. Braga, J. High Energy Phys. 03 (2014) 058.

[7] N. R. F. Braga, L. F. Ferreira, and A. Vega, Phys. Lett. B 774, 476 (2017); N. R. F. Braga and L. F. Ferreira, Phys. Lett. B 783, 186 (2018); N. R. F. Braga, L. F. Ferreira, and R. Da Rocha, Phys. Lett. B 787, 16 (2018).

[8] S. P. Bartz and T. Jacobson, Phys. Rev. D 94, 075022 (2016).

[9] A. Vega and A. Ibanez, Eur. Phys. J. A 53, 217 (2017).

[10] A. Vega and M. A. Martin Contreras, arXiv:1808.09096.

[11] S. S. Afonin and A. D. Katanaeva, Eur. Phys. J. C 74, 3124 (2014); Phys. Rev. D 98, 114027 (2018).

[12] S. S. Afonin and I. V. Pusenkov, Eur. Phys. J. C 76, 342 (2016).

[13] J. Chen, S. He, M. Huang, and D. Li, J. High Energy Phys. 01 (2019) 165.

[14] M. Cheng et al., Phys. Rev. D 74, 054507 (2006).

[15] J. Gasser and H. Leutwyler, Phys. Lett. B 184, 83 (1987).
[16] H. Leutwyler, Nucl. Phys. B, Proc. Suppl. 4, 248 (1988); P. Gerber and H. Leutwyler, Nucl. Phys. B321, 387 (1989).

[17] S. Weinberg, Physica (Amsterdam) 96A, 327 (1979); J. Gasser and H. Leutwyler, Ann. Phys. (N.Y.) 158, 142 (1984).

[18] A. Karch, E. Katz, D. T. Son, and M. A. Stephanov, Phys. Rev. D 74, 015005 (2006).

[19] S. J. Brodsky and G. F. de Teramond, Phys. Rev. Lett. 96, 201601 (2006); Phys. Rev. D 77, 056007 (2008).

[20] T. Branz, T. Gutsche, V. E. Lyubovitskij, I. Schmidt, and A. Vega, Phys. Rev. D 82, 074022 (2010).

[21] T. Gutsche, V.E. Lyubovitskij, I. Schmidt, and A. Vega, Phys. Rev. D 85, 076003 (2012).

[22] T. Gutsche, V. E. Lyubovitskij, I. Schmidt, and A. Vega, Phys. Rev. D 87, 056001 (2013).

[23] T. Gutsche, V. E. Lyubovitskij, and I. Schmidt, Phys. Rev. D 96, 034030 (2017).

[24] D. Toublan, Phys. Rev. D 56, 5629 (1997).

[25] T. Regge and J. A. Wheeler, Phys. Rev. 108, 1063 (1957).

[26] G. T. Horowitz and V. E. Hubeny, Phys. Rev. D 62, 024027 (2000).

[27] H. R. Grigoryan and A. V. Radyushkin, Phys. Rev. D 76, 095007 (2007).

[28] A. Ayala, C. A. Dominguez, and M. Loewe, Adv. High Energy Phys. 2017, 1 (2017).

[29] C. A. Dominguez, M. Loewe, and J. S. Rozowsky, Phys. Lett. B 335, 506 (1994). 